\title{
La cytométrie en flux : analyse prospective de son évolution à court et long terme
}

\begin{abstract}
Une initiative du Centre de prospective et d'études du ministère de la Recherche et de la Technologie sur l'évolution de la cytométrie en flux, menée par monsieur L.D. Nguyen, a contribué à la réalisation de ce manuscrit.
\end{abstract}

Initialement développée pour le dénombrement des cellules en suspension et l'évaluation de leur taille, la cytométrie en flux est rapidement devenue une technique d'analyse et de quantification de différents paramètres obtenus à partir de signaux émis par une cellule.

\section{- Principe (figure 1)}

Deux principes fondamentaux caractérisent la cytométrie en flux :

- le guidage des cellules en suspension à l'aide d'un flux laminaire liquide assurant leur défilement en "file indienne" devant un système d'illumination ;

- l'utilisation d'un système d'illumination et de détection suffisamment performant pour effectuer l'analyse d'une cellule donnée dans un temps très court, de l'ordre de quelques microsecondes.

Le principe conventionnel de fonctionnement consiste à guider et aligner (par surpression ou aspiration) dans une gaine liquide, de manière hydrodynamique et à vitesse constante, une suspension de particules isolées (cellules, constituants subcellulaires: noyaux, chromosomes, mitochondries... ou microorganismes). Chaque particule est éclairée individuellement, généralement dans une chambre de mesure, par une source lumineuse (laser, lampe à arc). Elle émet alors différents signaux optiques recueillis réel ou différé par un système de gestion informatique approprié, en raison de la vitesse de défilement (de 100 à 2000 particules/seconde). Les signaux correspondant à l'absorption, la diffusion (résultante des phénomènes de réflexion, réfraction et diffraction) et l'émission de fluorescence peuvent être quantifiés. Ceux-ci sont séparés de la lumière excitatrice par des miroirs et filtres optiques, puis transmis vers des détecteurs optroniques dont le rôle est de les convertir et de les amplifier en courants d'amplitude proportionnelle à la quantité de lumière reçue. Ces signaux sont ensuite analysés, accumulés et visualisés sous la forme de distributions monoparamétriques (figure 2) ou biparamétriques.

La conception de la cytométrie en flux permet également le tri physique de cellules ou constituants cellulaires ayant des propriétés spécifiques au sein d'une population. A la sortie de la zone de mesure, un transducteur piézo-électrique fractionne le flux liquide en gouttelettes. Chaque gouttelette contenant une particule intéressante est chargée électriquement (positivement ou négativement) à l'instant de la segmentation de la gaine liquide par un anneau de charge. Elle est ensuite déviée par un champ électrique créé par deux plaques portées à des potentiels opposés et recueillie dans un collecteur où sont regroupées les particules ayant des propriétés communes.

\section{- Évolution à court terme}

La cytométrie en flux est devenue un instrument essentiel, quotidiennement utilisé en biologie et en recherche biomédicale [1]. Cette technologie fournit un lien étroit entre les expérimentations au niveau moléculaire et génomique et celles réalisées au niveau des tissus et des organismes. Elle est également bien adaptée à la mise en évidence de maladies impliquant des modifications quantitatives et non qualitatives. Les applications des analyses par cytométrie en flux pour le diagnostic clinique ont subi une période de mise en œuvre due, dans certains cas, à la surestimation de la validité des marqueurs tumoraux, à la sous-estimation de l'hétérogénéité tumorale, mais également à l'absence de standardisation et au coût de l'analyse. Compte tenu des progrès de la standardisation et d'une évolution vers des appareils destinés à la routine moins onéreux que ceux destinés à la recherche [2-5], la cytométrie en flux affirmera très probablement sa place dans un certain nombre de spécialités cliniques.

L'évolution à court terme concerne deux types d'appareillages: les analyseurs-trieurs et les analyseurs de routine.

L'évolution des analyseurs-trieurs repose fortement sur l'utilisateur ; elle sera vraisemblablement "à la carte " en fonction des intérêts des responsables et relativement réduite, compte tenu de l'hétérogénéité technologique du parc de ces appareils. L'évolution des analyseurs de routine sera différente et portera sur des spécialisations en fonction des applications à cibler (immunologie, cycle cellulaire...). Ces appareils sont souvent utilisés par des non-spécialistes ; leur souplesse est relativement faible, mais leur maintenance est également très réduite. En particulier, les progrès de l'optique et un marché moins 
limité doivent donner un nouvel essor aux sources lumineuses classiques (lampes à arc); leur disponibilité, leur faible coût, leur simplicité d'utilisation, leur spectre étendu et, surtout, les faibles risques liés à leur usage sont des atouts importants face aux sources laser. L'interprétation des analyses réalisées sur ce type d'appareils nécessitera évidemment une formation minimale des utilisateurs. A cet égard, il convient de mentionner une aide essentielle qu'apporteront des logiciels intégrés simples, conviviaux (ne nécessitant pas de connaissances informatiques particulières) et spécialisés. Le développement de systèmes experts, c'est-

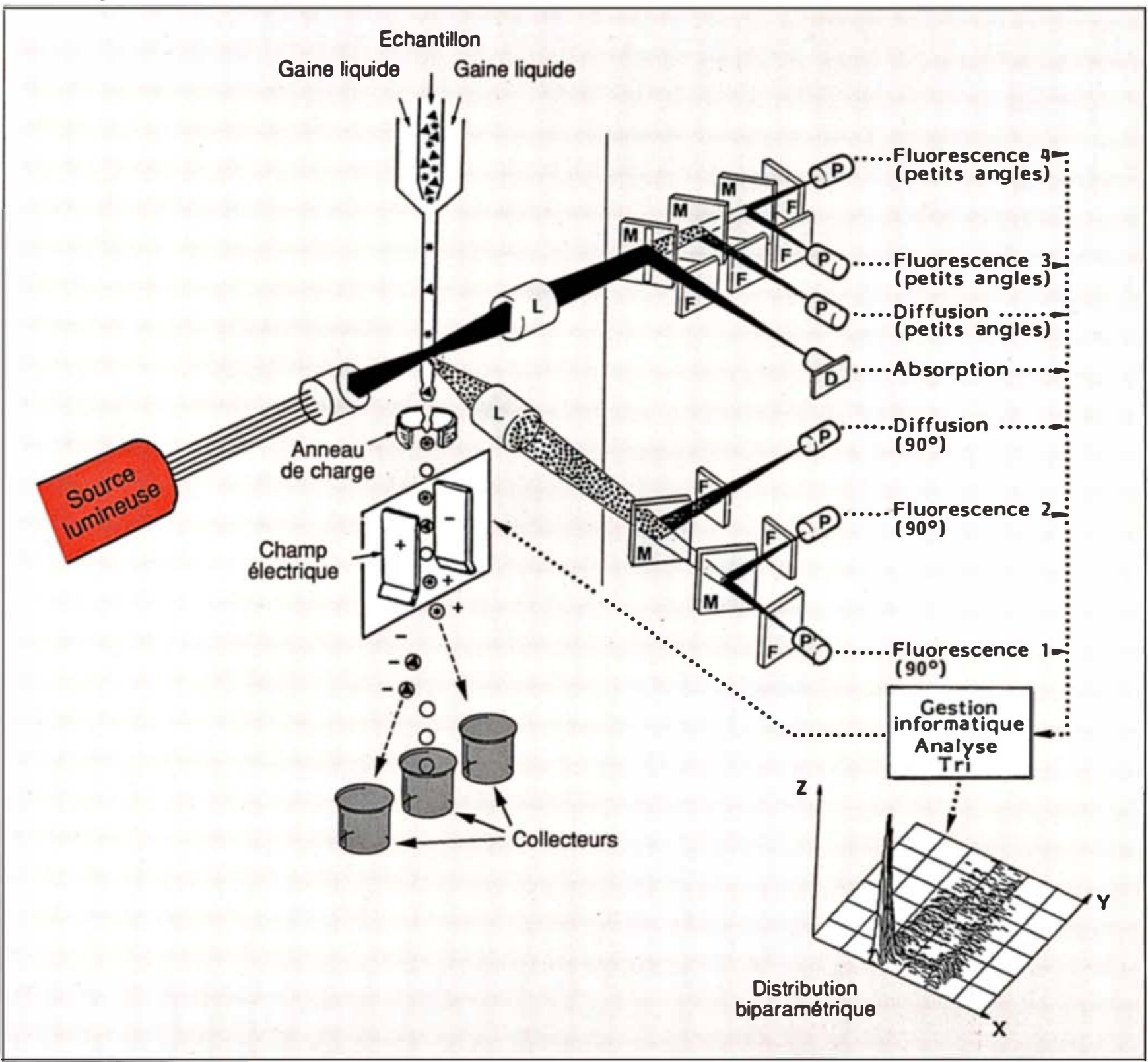

Figure 1. Principe général de fonctionnement d'un analyseur trieur de cellules. L'échantillon à analyser, constitué de particules en suspension et monodispersées, est entraîné par une gaine liquide. Chaque particule est éclairée par une source lumineuse (laser, lampe à arc). La particule induit une perturbation du signal lumineux incident (absorption, diffusion) ou émet des signaux lumineux (fluorescence). Ces signaux, sélectionnés par des miroirs et des filtres appropriés, sont recueillis par des photodétecteurs. Les paramètres peuvent être visualisés sous la forme de distributions mono- ou biparamétriques. La rapidité du traitement électronique permet le tri physique des particules par fractionnement de la gaine liquide en gouttelettes. Chaque gouttelette contenant une particule dont les paramètres ont été retenus est chargée et recueillie dans un collecteur. $D$ : photodiode ; $L$ : lentille convergente ; $F$ : filtre ; $M$ : miroir; $P$ : photomultiplieur. 
à-dire de réservoirs de connaissances, capables de stocker, d'exploiter correctement l'information et doués d'un pouvoir décisionnel, présentera une aide au diagnostic [6].

L'automatisme entraînera également une standardisation de l'utilisation des analyseurs par l'élaboration et l'adaptation de passeurs et de préparateurs d'échantillons ; cette évolution réduira également l'erreur humaine. Des réglages optiques asservis et gérés par un ordinateur, l'addition d'une référence interne, défini et reconnu par l'informatique, en permanence dans chaque échantillon, sont des facteurs qui assureront simultanément une optimalisation de l'environnement instrumental et biologique. Pertinence des systèmes, étalonnage, standardisation, lan-

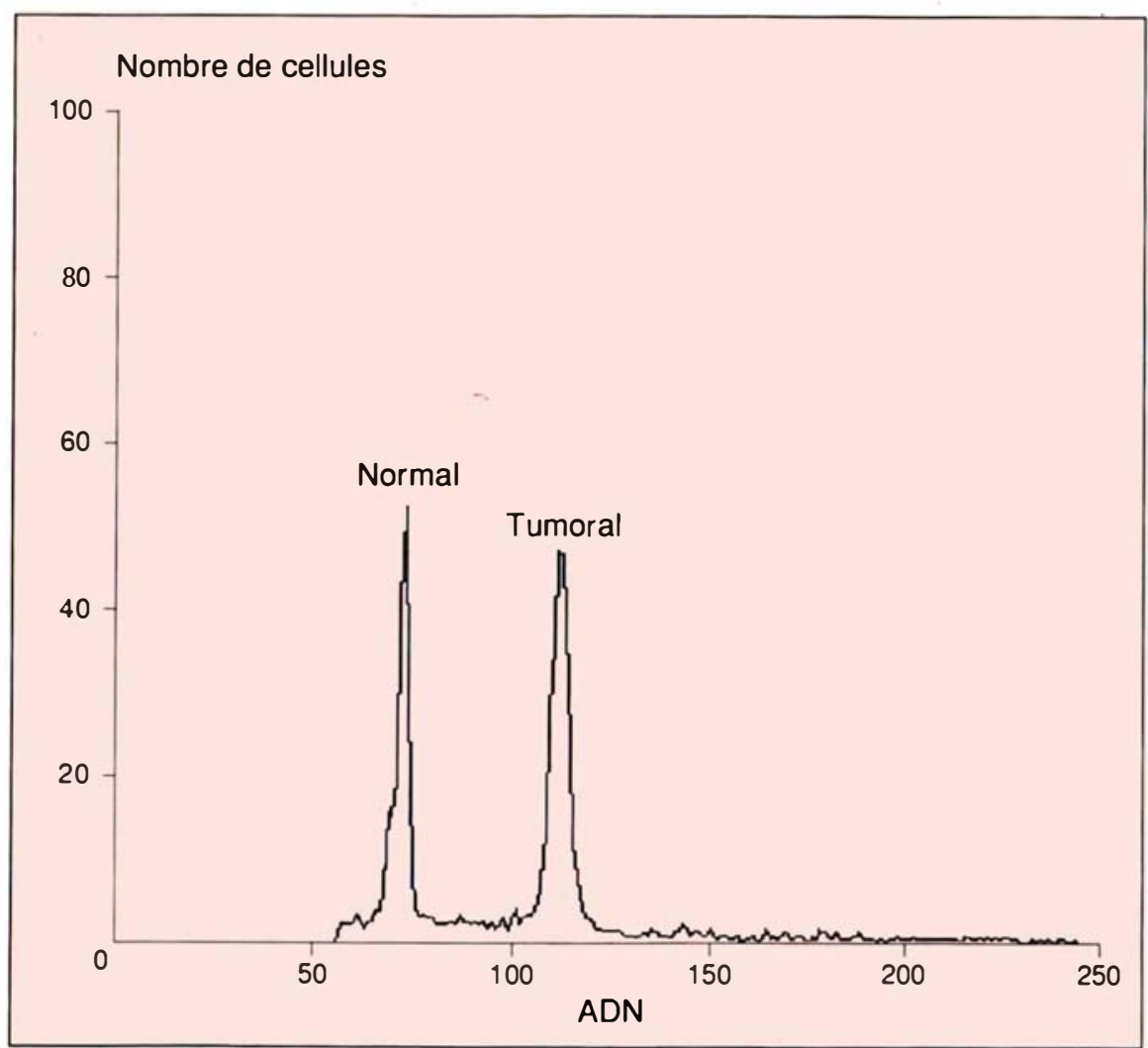

Figure 2. Histogramme de répartition de cellules d'une tumeur rectocolique selon leur quantité d'ADN (fluorescence verte, coloration par la mithramycine). Les cellules provenant d'un tissu tumoral (tumeur recto-colique) sont dissociées mécaniquement. Une coloration par un fluorochrome spécifique des bases C-G de l'ADN (la mithramycine) permet la mesure de la fluorescence émise en fonction du contenu en ADN. Les débris et éventuels agrégats cellulaires ont été éliminés par la mesure des diffusions. La représentation monoparamétrique obtenue révèle deux populations cellulaires de contenus en $A D N$ différents. La comparaison à des références permet d'identifier

gage commun entre biologistes et les populations normale et anormale (tumorale). teurs sont des termes clés qui présideront à une évolution positive de la cytométrie en flux [7].

\section{- Évolution à long terme}

Celle-ci dépend principalement des sondes biologiques, notamment dans le domaine des fluorochromes et substrats fluorogènes réunissant plusieurs critères essentiels : coefficients d'extinction élevés, rendement quantiques optimalisés, spectres d'excitation proches et spectres d'émission nettement séparés, simplicité de conjugaison aux sondes spécifiques [8]. développement de fluorochromes spécifiques de constituants cellulaires particuliers constitue, par ailleurs, une étape critique pour l'amélioration

equ

Xavier Ronot
École pratique des hautes études, Paris et
équipe de reconnaissance des formes et
microscopie quantitative, Cermo, université
Joseph-Fourier, BP 53X, 38041 Grenoble
Cedex, France.
José Pierrez
Eurocyt SA, 32 boulevard de Lyon, 67000
Strasbourg, France.

Xavier Ronot
École pratique des hautes études, Paris et
équipe de reconnaissance des formes et
microscopie quantitative, Cermo, université
Joseph-Fourier, BP 53X, 38041 Grenoble
Cedex, France.
José Pierrez
Eurocyt SA, 32 boulevard de Lyon, 67000
Strasbourg, France.

Xavier Ronot
École pratique des hautes études, Paris et
équipe de reconnaissance des formes et
microscopie quantitative, Cermo, université
Joseph-Fourier, BP $53 X, 38041$ Grenoble
Cedex, France.
José Pierrez
Eurocyt SA, 32 boulevard de Lyon, 67000
Strasbourg, France.

Xavier Ronot
École pratique des hautes études, Paris et
équipe de reconnaissance des formes et
microscopie quantitative, Cermo, université
Joseph-Fourier, BP $53 X, 38041$ Grenoble
Cedex, France.
José Pierrez
Eurocyt SA, 32 boulevard de Lyon, 67000
Strasbourg, France.

Xavier Ronot
École pratique des hautes études, Paris et
équipe de reconnaissance des formes et
microscopie quantitative, Cermo, université
Joseph-Fourier, BP $53 X, 38041$ Grenoble
Cedex, France.
José Pierrez
Eurocyt SA, 32 boulevard de Lyon, 67000
Strasbourg, France.

Xavier Ronot
École pratique des hautes études, Paris et
équipe de reconnaissance des formes et
microscopie quantitative, Cermo, université
Joseph-Fourier, BP $53 X, 38041$ Grenoble
Cedex, France.
José Pierrez
Eurocyt SA, 32 boulevard de Lyon, 67000
Strasbourg, France.

Xavier Ronot
École pratique des hautes études, Paris et
équipe de reconnaissance des formes et
microscopie quantitative, Cermo, université
Joseph-Fourier, BP $53 X, 38041$ Grenoble
Cedex, France.
José Pierrez
Eurocyt SA, 32 boulevard de Lyon, 67000
Strasbourg, France.

\section{Remerciements}

Les auteurs remercient le Dr André Burckel pour les critiques apportées lors de la rédaction de ce manuscrit.

\section{RÉFÉRENCES}

1. Ran DH, Fallon MA, Horan PK. Flow cytometry in the clinical laboratory. Clin Chim Acla 1988 ; $171 ; 125-74$.

2. Ashcroft RG. Future clinical role for flow cytometry. Cytometry 1988 ; suppl. : 85-8. 3. Hanson CA. Applications of flow cytometry in diagnostic pathology. Am J Clin Pathol 1989 ; 91 : 527-31.

4. Burger G. Cytometry, a possible tool for assessment of tumor response. Strahlenther Onk 1989; 165 : 291-3.

5. Darzynkiewicz Z. Flow cytometry in cytopathology : overview and perspectives. Anal Quant Cytol Histol $1988 ; 10$ : 459-61. 6. Bagwell CB. New horizons : expert system for flow cytometry. Cytometry 1988 ; suppl. : 89-93.

7. Harvath L. Quality control in clinical flow cytometry. Pathol Immunopathol Res $1988 ; 7: 338-44$

8. Lin TI, Jovanovic MV, Dowben RM. Nine new fluorescent probes. In : Salzman GC, ed. New Technologies in Cytometry. Washington : SPIE Proc 1989; 1063 : 133-41.

des applications existantes et le déve-
loppement d'applications nouvelles. Les méthodes de détection de la fluorescence nécessitent des perfectionnesignal/bruit du fluorochrome. En outre, les méthodes d'hybridation en une détection performante par cytométrie en flux de molécules présende sondes hautement spécifiques et le détection de cellules rares permettront une analyse des régulations cellulaires au niveau génétique, de ont amélioré la compréhension de l'immunorégulation au niveau cellulaire

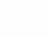

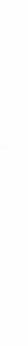

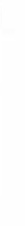

